\title{
Gastric cancer cell adhesion to laminin enhances acquired chemotherapeutic drug resistance mediated by MGr1-Ag/37LRP
}

\author{
LI SUN $^{1 *}$, LILI LIU ${ }^{2 *}$, XIANGQIANG LIU ${ }^{1}$, YAFANG WANG ${ }^{2}$, MENGBIN LI $^{1}$, LIPING YAO ${ }^{1}$, \\ JIANJUN YANG ${ }^{1}$, GENLIN JI ${ }^{3}$, CHANGCUN GUO ${ }^{1}$, YANGLIN PAN ${ }^{1}$, SHUHUI LIANG $^{1}$, \\ BIAOLUO WANG ${ }^{1}$, JIE DING $^{1}$, HONGWEI ZHANG $^{1}$ and YONGQUAN SHI ${ }^{1}$
}

\author{
${ }^{1}$ State Key Laboratory of Cancer Biology, Xijing Hospital of Digestive Diseases, Fourth Military Medical University, \\ Xi'an 710032; ${ }^{2}$ Department of Oncology, Tangdu Hospital, Fourth Military Medical University, Xi'an 710038; \\ ${ }^{3}$ Department of Anesthesiology, Xijing Hospital, Fourth Military Medical University, Xi'an 710032, P.R. China
}

Received February 10, 2014; Accepted March 22, 2014

DOI: $10.3892 /$ or.2014.3184

\begin{abstract}
Adhesion of cancer cells to the extracellular matrix (ECM) causes a novel acquired chemotherapeutic drug-resistant phenotype, referred to as cell adhesion-mediated drug resistance (CAM-DR). Our previous studies suggested that the adhesion molecule MGr1-Ag/37LRP may promote multidrug resistance in gastric cancer cells. Therefore, we investigated MGr1-Ag/37LRP binding-induced adhesion, and its role in CAM-DR. Initial studies revealed that, after adhesion to the ECM, the multidrug-resistant gastric cancer cell lines SGC7901/VCR and SGC7901/ADR showed significantly higher mean adhesive cell numbers than non-resistant SGC7901 cells. We then investigated expression of MGr1$\mathrm{Ag} / 37 \mathrm{LRP}$ in gastric cancer cells adhering to laminin. Western blotting, RT-PCR and dual-luciferase reporter assays showed that laminin induced MGr1-Ag/37LRP expression and activity. In vitro and in vivo assays revealed that small interfering RNA against MGr1-Ag/37LRP significantly reduced CAM-DR in SGC7901/VCR cells. In vivo and in vitro analyses revealed that binding of MGrl-Ag/37LRP decreased intracellular drug accumulation by increasing P-glycoprotein and multidrug-associated protein expression, and inhibited drug-induced apoptosis by regulating Bcl-2 and Bax expression. These results indicate that MGr1-Ag/37LRP contributes to laminin-mediated CAM-DR in gastric cancer cells, and is a potentially effective target for reversing this phenomenon in gastric cancer.
\end{abstract}

Correspondence to: Professor Yongquan Shi or Professor Hongwei Zhang, State Key Laboratory of Cancer Biology, Institute of Digestive Diseases, Xijing Hospital, Fourth Military Medical University, No. 15 Changle West Road, Xi'an 710032, P.R. China

E-mail: shiyquan@fmmu.edu.cn

E-mail: zhanghw@fmmu.edu.cn

${ }^{*}$ Contributed equally

Key words: MGrl-Ag/37LRP, CAM-DR, gastric cancer, laminin, cell adhesion

\section{Introduction}

The resistance of gastric cancer cells to multiple chemotherapeutic agents remains a major therapeutic obstacle. MGrl-Ag is an upregulated protein in drug-resistant SGC7901/VCR cells. Using SGC7901/VCR cells as the immunogen, we prepared a monoclonal antibody against MGr1-Ag named MGrl (1), and obtained the gene MGr1-Ag (GenBank AF503367) by screening a cDNA library with the MGr1 monoclonal antibody. Sequence analysis revealed that MGrl-Ag is identical to the human $37-\mathrm{kDa}$ laminin receptor precursor protein (37LRP) (2). Further study suggested that MGr1-Ag/37LRP may promote MDR in gastric cancer cells by decreasing intracellular drug accumulation and inhibiting drug-induced apoptosis (3). However, the exact mechanism of the contribution of MGr1-Ag/37LRP to MDR in gastric cancer remains unknown.

The extracellular matrix (ECM) of cancer cells profoundly influences major malignant phenotypes, including oncogenesis, progression and apoptosis (4). Adhesion confers a novel acquired chemotherapeutic drug-resistant phenotype referred to as cell adhesion-mediated drug resistance (CAM-DR) (5). Laminin (LN) and collagen IV (COL IV) are natural basement membrane components that constitute a specific ECM that maintains malignant phenotypes in gastric adenocarcinoma cells $(6,7)$. MGr1-Ag/37LRP directly correlates with tumor growth and proliferation as a laminin receptor (8). Therefore, we hypothesized that MGrl-Ag/37LRP binding-induced adhesion and the binding-initiated intracellular signaling pathways participate in protecting gastric cancer cells from a number of apoptotic stimuli caused by chemotherapeutic drugs.

In the present study, we investigated whether MGr1$\mathrm{Ag} / 37 \mathrm{LRP}$ binding-induced adhesion participates in protecting gastric cancer cells from apoptotic stimuli caused by chemotherapeutic drugs. We found that MGr1-Ag/37LRP binding decreased intracellular drug accumulation by inhibiting the expression of P-glycoprotein (P-gp) and multidrug resistanceassociated protein (MRP), and inhibited drug-induced apoptosis through regulation of $\mathrm{Bcl}-2$ and Bax expression. Sensitivity to chemotherapeutic drugs in xenografts was significantly enhanced by inhibiting MGrl-Ag/37LRP 
expression. These studies aimed to characterize the role, and the molecular mechanisms of the effects of MGr1-Ag/37LRP on CAM-DR in gastric cancer cells.

\section{Materials and methods}

Cell lines and cell culture. The human gastric adenocarcinoma cell line SGC7901 was a gift from the Academy of Military Medical Science (Beijing, China). We previously generated and characterized the MDR gastric cancer cell variants SGC7901/VCR and SGC7901/ADR (9). All cell lines were maintained in RPMI-1640 supplemented with 10\% heatinactivated fetal calf serum (both from Gibco, Grand Island, NY, USA) and antibiotics, at $37^{\circ} \mathrm{C}$ in a humidified atmosphere of $5 \% \mathrm{CO}_{2}$ and $95 \%$ air. To maintain the drug-resistant phenotype, SGC7901/VCR cells received vincristine (VCR), and SGC7901/ADR cells received adriamycin (ADR) at $1 \mu \mathrm{g} / \mathrm{ml}$. Two weeks before assessing MDR in gastric cancer cells and the transfected cells, VCR and ADR treatment was ceased, to eliminate drug exposure effects.

Plasmids and transfection. The sense expression vector pcDNA3.1/MGr1 and the siRNA vector of MGr1-Ag/37LRP were constructed previously in our laboratory $(3,10)$. Vectors pGL3-MGr1L and pGL3-MGr1S contain promoter fragments for nucleotides -1600 to +964 , and -292 to +964 relative to transcription start constructed previously in our laboratory (11). Drug-sensitive SGC7901 cells were transfected with the sense vector pcDNA3.1/MGr1 to generate line SGC7901-MGr1, or with the control vector pcDNA3.1 to generate SGC7901-pc. Drug-resistant SGC7901/VCR cell line was transfected with the siRNA targeting MGr1-Ag/37LRP and named SGC7901/VCR-siMGr1, or the control vector pSilenser and named SGC7901/VCR-ps. Cell transfection was carried out with Lipofectamine 2000 (Invitrogen, Carlsbad, CA, USA) according to the manufacturer's protocol. For transient transfection, cells were harvested after $48 \mathrm{~h}$; for stable transfecion, $\mathrm{G} 418(400 \mu \mathrm{g} / \mathrm{ml})$ was added after $24 \mathrm{~h}$, and mixed clones were screened and expanded for an additional 6 weeks.

Western blotting and immunohistochemistry. Whole cells pretreated as indicated, or harvested tumor tissue, were lysed on ice for $30 \mathrm{~min}$ in lysis buffer $(10 \mathrm{mM}$ Tris, $\mathrm{pH} 8.0,1 \mathrm{mM}$ EDTA, $400 \mathrm{mM} \mathrm{NaCl}, 10 \%$ glycerol, $0.5 \% \mathrm{NP}-40,5 \mathrm{mM}$ sodium fluoride, $0.1 \mathrm{mM}$ phenylmethylsulfonyl fluoride, $1 \mathrm{mM}$ dithiothreitol). Equal amounts of protein $(25 \mu \mathrm{g})$ were analyzed by western blotting with anti-P-gp, anti-MRP, anti-Bcl-2, antiBax (Santa Cruz Biotechnology Inc.), anti-MGrl-Ag [prepared by our laboratory (12)], or anti- $\beta$-actin (Sigma, USA) for a 3-h incubation. Blots were washed, and a species-matched peroxidase-conjugated secondary antibody was added $(1: 2,000)$. Labeled bands from the washed blots were detected with an ECL kit (Amersham).

Tissues were dewaxed in xylene, washed in $96 \% \mathrm{vol} / \mathrm{vol}$ ethanol, and incubated for $30 \mathrm{~min}$ in $0.3 \%$ hydrogen peroxide before washing in phosphate-buffered saline (PBS). Blocking was for $30 \mathrm{~min}$ with $1 \%$ bovine serum albumin (BSA) at room temperature, followed by incubation for $120 \mathrm{~min}$ at room temperature with the MGr1-Ag/37LRP antibody in PBST $(0.01 \%)$ supplemented with $10 \%$ milk powder. Samples were washed three times with PBST (0.01\% Tween) and incubated with the secondary antibody for $30 \mathrm{~min}$, before processing with the Vectastain Elite ABC kit according to the manufacturer's instructions (Vector Laboratories) prior to digital photography on a Nikon Eclipse E600 microscope with a Spot RT slider camera and imaging software (Imsol Imaging Solutions).

Semi-quantitive reverse transcription ( $R T)$ - $P C R$ analysis. Total RNA was extracted using TRIzol reagent (Invitrogen) according to the manufacturer's recommendations. RT-PCR analysis of mRNA levels was performed using primers specific for MGr1-Ag (yielding $463 \mathrm{bp}$ ) forward primer, 5'-GCTGGACGATAGCTTGGA-3' and reverse primer, 5'-GATGACAGATAGCTGGTG-3'; for $\beta$-actin (yielding $287 \mathrm{bp}$ ) forward primer, 5'-AGCGGGAAATCGTGCGTG-3' and reverse primer, 5'-CAGGGTACATGGTGGTGCC-3'. PCR consisted of $94^{\circ} \mathrm{C}$ for $4 \mathrm{~min}, 30$ cycles of $94^{\circ} \mathrm{C}$ for $50 \mathrm{sec}$, $60^{\circ} \mathrm{C}$ for $50 \mathrm{sec}$ and $70^{\circ} \mathrm{C}$ for $30 \mathrm{sec}$, in a Touchgene Gradient thermal cycler (Techne, Cambridge, UK). PCR products were analyzed by agarose gel electrophoresis to determine PCR quality.

Cell adhesion assay. Gastric cancer cell adherence to ECM components LN (LAMB1; Sigma) and COL IV, or to the BSA control was determined in 24-well plates as previously described (13). The plate surface was covered with $1 \mu \mathrm{g} / \mathrm{cm}^{2}$ $\mathrm{LN}, 1 \mu \mathrm{g} / \mathrm{cm}^{2}$ COL IV or $0.4 \mu \mathrm{g} / \mathrm{cm}^{2}$ BSA, incubated for $2 \mathrm{~h}$, and the supernatant was removed. A suspension of tumor cells $\left(1 \times 10^{5} / \mathrm{ml}, 0.5 \mathrm{ml}\right)$ was transferred into the covered wells. After $0.5,1,2$ or $4 \mathrm{~h}$ of incubation at $37^{\circ} \mathrm{C}$, the adhesive cells were washed with PBS twice and counted under a microscope at a x200 magnification in 10 random fields/well. Each experiment was performed in triplicate.

In vitro drug sensitivity assay. For the colony-formation assays, gastric cancer cells in log phase were harvested and plated into $35-\mathrm{mm}$ culture plates $\left(1 \times 10^{3}\right.$ cells/well $)$ covered with ECM components or BSA. In some cases, cells were preincubated with different concentrations of MGrl-AG/37LRP siRNA. After overnight incubation at $37^{\circ} \mathrm{C}$ for adhesion, VCR or 5-fluorouracil $(5-\mathrm{Fu})$ was added and the incubation was continued for $24 \mathrm{~h}$. Plates were washed twice with serum-free RPMI-1640, and grown in complete culture medium (RPMI1640 supplemented with $10 \%$ heat-inactivated fetal calf serum and antibiotics), for 10 days. The resulting colonies were stained with Coomassie Brilliant Blue, and visible colonies were counted. The concentration of drug that caused a $50 \%$ reduction in the number of colonies $\left(\mathrm{IC}_{50}\right)$ was calculated using SPSS 11.0 software (Chicago, IL, USA).

Fluorescence intensity assay for intracellular ADR. The fluorescence intensity of intracellular ADR was determined by flow cytometry, as described previously (14). In brief, gastric cancer cells in log-phase were seeded into 6-well plates $\left(1 \times 10^{6}\right.$ cells/ well) coated with ECM components or BSA and cultured overnight at $37^{\circ} \mathrm{C}$. After addition of ADR to a final concentration of $5 \mathrm{mg} / \mathrm{l}$, cells were cultured for $1 \mathrm{~h}$, then trypsinized and harvested to detect ADR accumulation by flow cytometry (Coulter, Miami, FL, USA) with an excitation wavelength of 
$488 \mathrm{~nm}$ and an emission wavelength of $575 \mathrm{~nm}$. The ADR release index was calculated according to the formula: Release index $=($ accumulation value - retention value $)$ /accumulation value.

Annexin V/propidium iodide staining. The apoptotic index (AI) of gastric cancer cells was calculated as the number of apoptotic cells detected by flow cytometry. In brief, cells in log phase were plated into 6 -well plates $\left(1 \times 10^{6}\right.$ cells/well $)$ coated with ECM components or BSA and cultured overnight at $37^{\circ} \mathrm{C}$. VCR was added to a final concentration of $0.3-0.6 \mathrm{mg} / \mathrm{l}$, and culturing was continued for 36-72 h. Annexin V-FITC (5 $\mu \mathrm{l})$ was added to the cells, and the mean fluorescence intensity of Annexin V-FITC/PI was determined by flow cytometry as described in the Clontech protocol (Palo Alto, CA, USA). AI was calculated as the mean fluorescence intensity.

Dual luciferase reporter assay. SGC7901 cells were plated at $3 \times 10^{5}$ cells $/ 35-\mathrm{mm}$ dish coated $\sim 12 \mathrm{~h}$ before transfection with $0.25,0.50,0.75,1.00$ or $1.50 \mu \mathrm{g} / \mathrm{cm}^{2}$ of LN. Transient transfections used Lipofectamine 2000 reagent (Invitrogen) and $1.0 \mu \mathrm{g}$ of pGL3-MGr1L or pGL3-MGr1S, co-transfected with $0.1 \mu \mathrm{g}$ of phRL-TK vector (Promega) as an internal control. After $48 \mathrm{~h}$, the transfected cells were harvested, lysed, centrifuged to pellet the debri, and used for luciferase assays. Luciferase activity was measured as chemiluminescence, using a luminometer (PerkinElmer) and the Dual-Luciferase reporter assay system (Promega), according to the manufacturer's protocol. All transfections were performed in triplicate.

Assessment of in vivo tumor growth. Approximately $1 \times 10^{6}$ SGC7901/VCR cells were inoculated subcutaneously with $0.1 \mathrm{ml}$ of Matrigel (Sigma-Aldrich) in the flank region of 6 - to 8-week-old male athymic nude mice (Experimental Animal Center, FMMU, China) using a 27-gauge needle under halothane anesthesia. When tumors reached $100 \pm 20 \mathrm{~mm}^{3}$, usually 3-4 weeks after injection, mice were randomly selected for treatment with MGr1-Ag/37LRP siRNA or scrambled siRNA oligonucleotide, administered as $50 \mu \mathrm{l}$ of $1 \mu \mathrm{g} / \mu 1$ siRNA, or control PBS was administrated once every three days by intratumoral injection for 36 days (15). From day 7 to 14 , and 28 to $35,0.6 \mathrm{mg} / \mathrm{kg}$ of VCR was administered via tail vein injection (16). Tumor volume measurements were performed once every four days and calculated by the formula: Tumor volume $=$ length $\mathrm{x}$ width $\mathrm{x}$ depth $\mathrm{x} 0.5236$ (17). Data are expressed as average tumor volume levels \pm standard error (SE). All animal procedures were performed according to the guidelines of the Chinese Council on Animal Care, and with appropriate institutional certification.

Half of the transplanted tumors in each group were dissected and fixed in formalin for immunohistochemical studies, and the other half were immediately harvested in cold isopentane, frozen in liquid nitrogen, and kept at $-80^{\circ} \mathrm{C}$ for western blot analyses. Formalin-fixed tissues were processed into $5-\mu \mathrm{m}$ sections of formalin-fixed paraffin-embedded specimens for immunohistochemistry.

Statistical analysis. Each experiment was repeated at least three times. Bands from western blot analyses or RT-PCR were quantified by Quantity One software (Bio-Rad). Relative protein or mRNA levels were calculated relative to $\beta$-actin. Numerical data are presented as the means \pm standard error of the mean (SEM). Analysis of variance (ANOVA) was used to compare differences between experimental groups. LSD t-test was used for multiple comparisons. All statistical analysis was carried out with SPSS 11.0 software (Chicago, IL, USA). A $\mathrm{p}$-value $\leq 0.05$ was considered to indicate a statistically significant result.

\section{Results}

MDR of gastric cancer cells is enhanced by ECM adhesion. To gain insight into the relationship between the adhesive properties and drug resistance of gastric cancer, we compared the adhesive potential between the MDR variants SGC7901/VCR and SGC7901/ADR with the parental cell line SGC7901, after adhesion to the ECM components. As shown in Fig. 1, The MDR cells showed a significantly increased mean adhesion cell number than the SGC7901 cells after adhesion to both ECM and the BSA (Fig. 1A). This indicated that SGC7901/ VCR and SGC7901/ADR cells exhibited a relatively high adhesive potential to ECM.

As shown by colony-forming assays, SGC7901/VCR and SGC7901/ADR cells had significantly increased $\mathrm{IC}_{50}$ values for VCR or 5-Fu after adhesion to ECM components, compared to $\mathrm{IC}_{50}$ values for these agents in cells following adhesion to BSA (Fig. 1B). Similarly, after adhesion to ECM components, SGC7901/VCR and SGC7901/ADR cells showed significantly decreased AI values compared to the control, and had decreased ADR accumulation and retention, as well as increased release indices (Fig. 1C and D).

We next examined the expression of P-gp, MRP, Bcl-2 and Bax in both MDR and drug-sensitive gastric cancer cells after adhesion to ECM components. Western blot analysis showed that in the MDR gastric cancer SGC7901/VCR and SGC7901/ADR cells following adhesion to laminin, significantly increased expression of P-gp, MRP and Bcl-2 and decreased Bax expression were observed when compared to the drug-sensitive SGC7901 cells (Fig. 2). These results indicated that cell adhesion to ECM enhanced the MDR phenotype of the gastric cancer cell line SGC7901 by decreasing intracellular drug accumulation and by inhibiting drug-induced apoptosis, by regulating P-gp, MRP, Bcl-2 and Bax expression.

Cell adhesion to LN upregulates MGrl-Ag/37LRP expression in vitro. It has been reported that binding of $\mathrm{LN}$ by cell-surface LN receptors induces synthesis of 37LRP in melanoma cells, resulting in increased delivery of LN-binding proteins to the cell surface, and potentiating attachment to the basement membrane during invasion and metastasis (18). We investigated whether LN upregulates MGr1-Ag/37LRP expression by examining mRNA and protein levels and transcriptional activity by using reporter assays with MGr1 promoter-containing plasmids in gastric cancer cells. As shown in Fig. 3, using LN as an adhesion substrate significantly increased mRNA and protein in a dose-dependent manner. Luciferase reporter constructs with MGr1 promoter fragments of $2564 \mathrm{bp}$ (pGL3-2564-Luc, nucleotides -1600 to +964), or 1256 bp (pGL3-1256-Luc, nucleotides -292 to +964) were used to determine induction by LN. As shown in Fig. 3C, SGC7901 
A

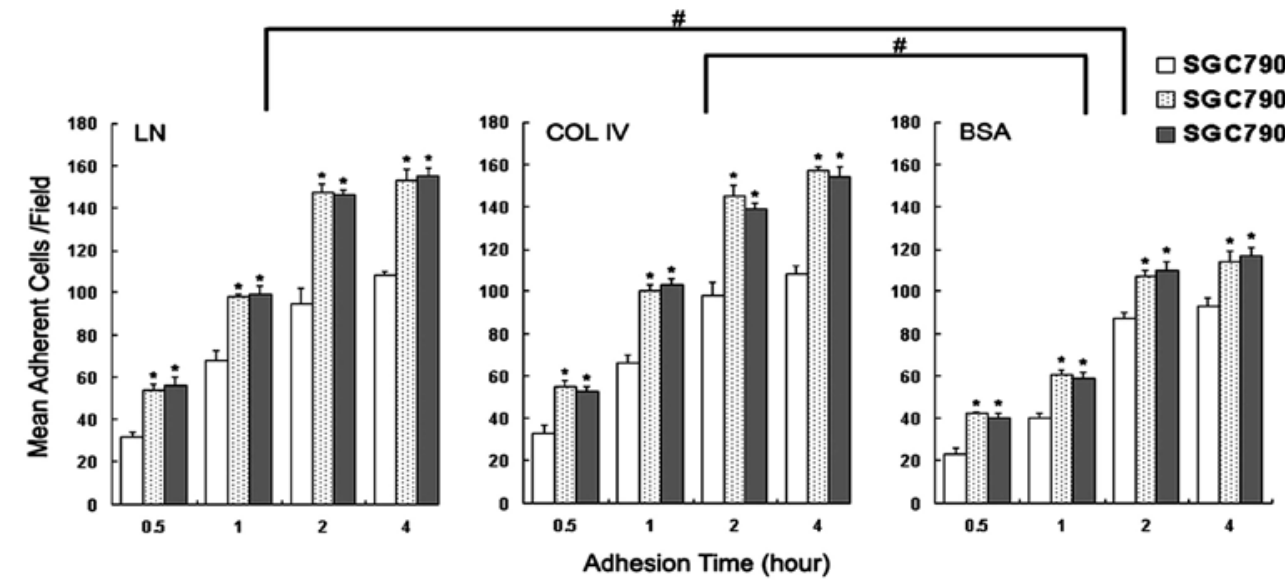

B

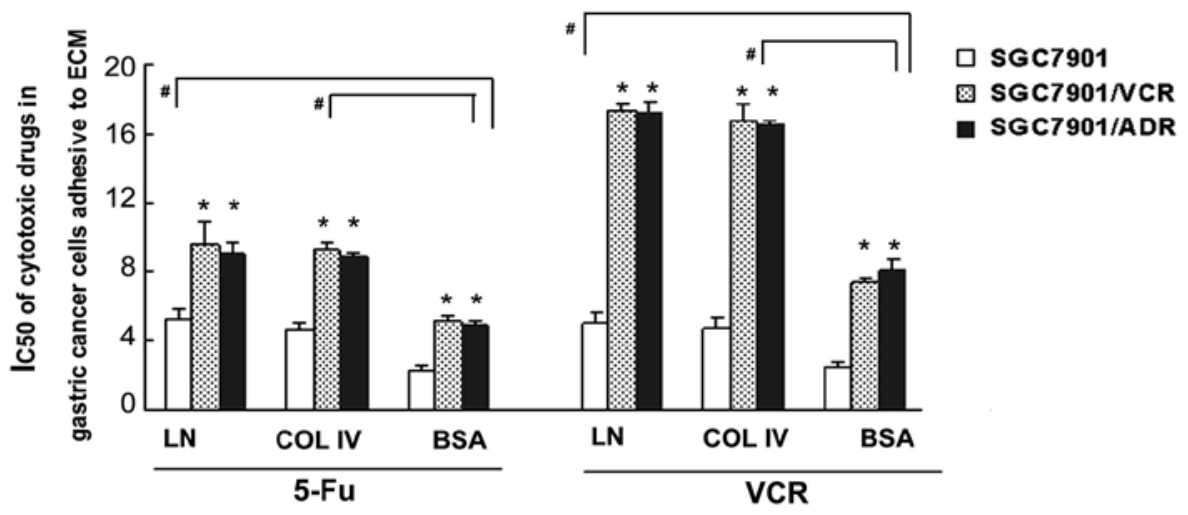

C

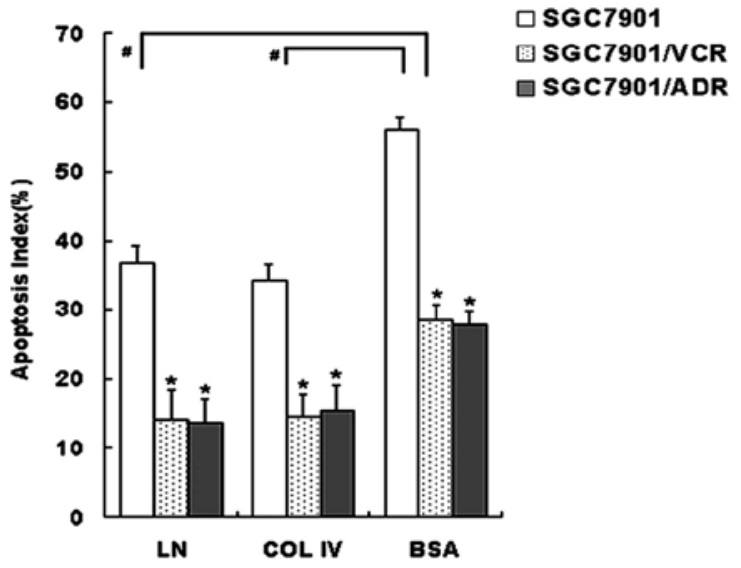

D

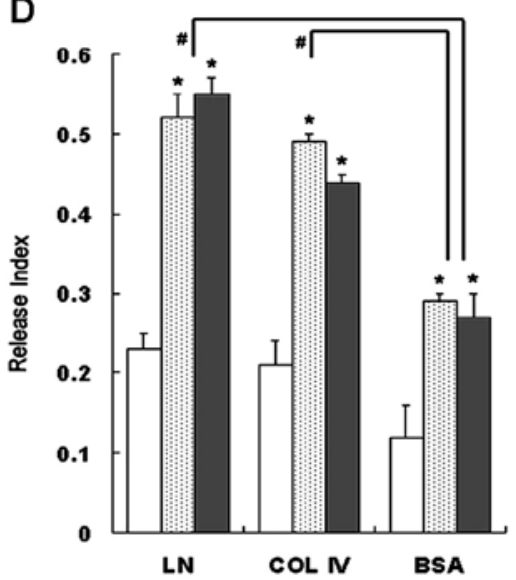

Figure 1. Phenotypic characterization of drug-sensitive and MDR gastric cancer cells after adhesion to ECM components. (A) Cells attached to ECM components were counted microscopically after $0.5,1,2$ or $4 \mathrm{~h}$ of adhesion. (B) Different gastric cancer cell lines were evaluated by colony-forming assay to calculate $\mathrm{IC}_{50}$. (C) Apoptotic indices of gastric cancer cells treated with VCR, as detected by flow cytometry. (D) ADR release indices of gastric cancer cells calculated by ADR accumulation and retention, as detected by flow cytometry. " $\mathrm{p}<0.05$ vs. SGC7901 adhering to ECM components and BSA control. "p $<0.05$, vs. adhering to BSA. ECM, extracellular matrix; ADR, adriamycin; BSA, bovine serum albumin.

cells treated with LN and transfected with pGL3-2564-Luc showed a significant, dose-dependent increase in luciferase activity over cells treated with the BSA control $(\mathrm{p}<0.05)$. The plasmid pGL3-1256-Luc showed no alteration in luciferase activity over the BSA control. These results revealed that LN induced MGrl-Ag expression at the transcriptional level, and the promoter nucleotides -1600 to -292 were essential for this effect.
Establishment of forced and siRNA expression of MGrl-Ag by stable transfection. We previously reported that overexpression of MGrl-Ag could prompt drug resistance in gastric cancer. To study the functional role of MGr1-Ag/37LRP in MDR in gastric cancer after adhesion to ECM, an MGr1-Ag/37LRP sense vector and an siRNA vector were used to upregulate and downregulate MGr1-Ag/37LRP. As shown by western blotting and RT-PCR analysis (Fig. 4), treatment of SGC7901 
A

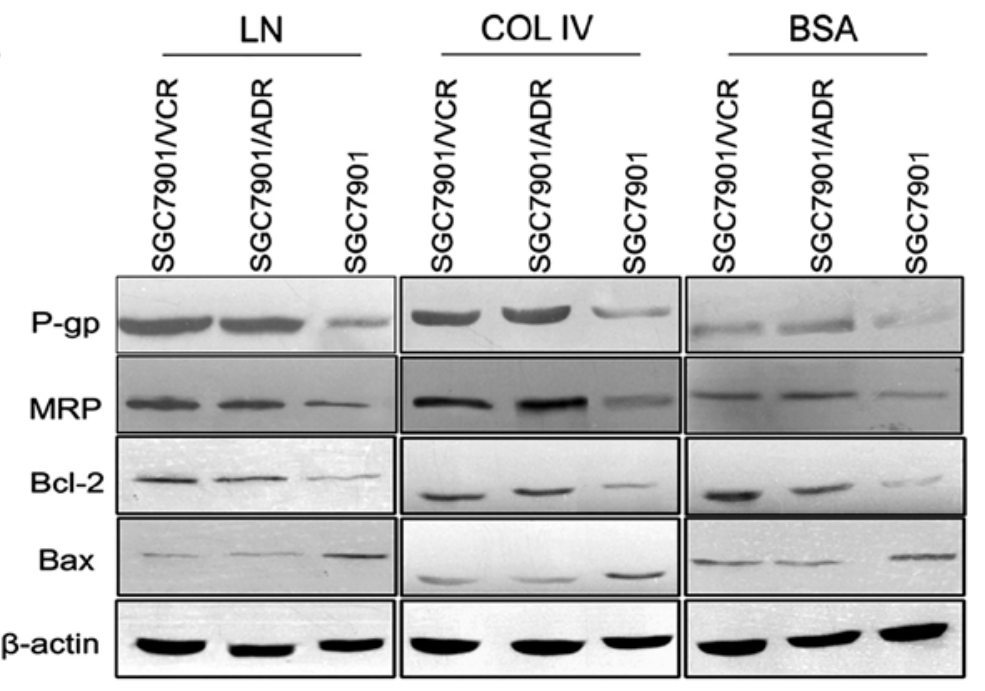

B

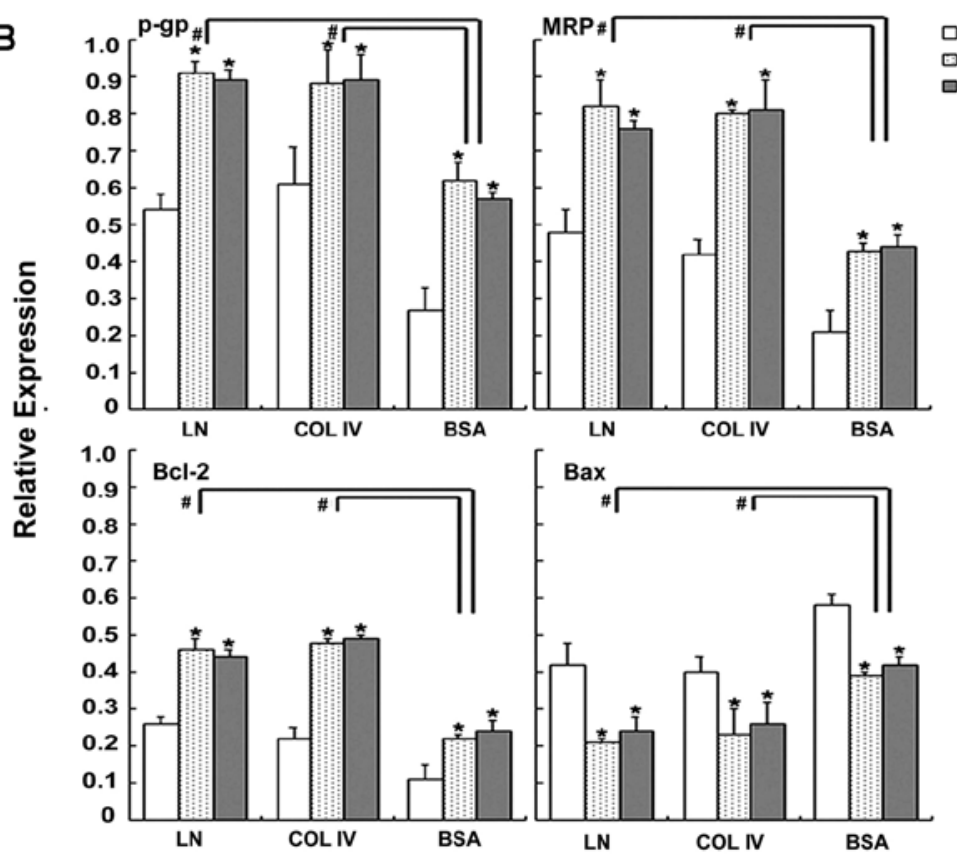

Figure 2. P-gp, MRP, Bcl-2 and Bax expression of gastric cancer cells adhering to ECM. (A) Expression of P-gp, MRP, Bcl-2 and Bax evaluated by western blotting. (B) Relative expression of P-gp, MRP, Bcl-2 and Bax. Signals were quantified by densitometric scanning. Data are expression relative to $\beta$-actin. "p $<0.05$ vs. SGC7901 adhering to ECM components or BSA control. ${ }^{*} \mathrm{p}<0.05$ vs. adhering to BSA. P-gp, P-glycoprotein; MRP, multidrug resistance-associated protein; ECM, extracellular matrix; BSA, bovine serum albumin.

cells with the MGrl-Ag/37LRP expression vector significantly induced MGr1-Ag/37LRP expression at the protein and mRNA levels (Fig. 4A and B, lane 1). SGC7901/VCR cells transfected with the MGr1-Ag/37LRP siRNA vector had significantly reduced protein and mRNA levels (Fig. 4A and B, lane 4), while a control vector with a scrambled oligonucleotide did not significantly suppress expression.

CAM-DR phenotype of gastric cancer cells is enhanced by MGrl-Ag/37LRP upregulation in vitro. To investigate the role of MGr1-Ag/37LRP in gastric cancer CAM-DR, cell adhesion assays, in vitro drug sensitivity assays, fluorescence intensity assays for intracellular ADR, Annexin V/PI staining, and western blotting were utilized to determine the MDR phenotype of gastric cancer cells with upregulation or down- regulation of MGrl-Ag/37LRP, after adhesion to LN, COL IV, or control BSA (Fig. 4C-E). Under the same conditions, SGC7901/VCR-siMGr1 cells showed significantly decreased mean adhesion cell numbers compared to SGC7901/VCR or SGC7901/VCR-ps cells. Adhesion to LN caused a significant increase in SGC7901-MGr1 cell adhesion, over adhesion to COL IV (p<0.05, Fig. 4C-E).

Significantly increased $\mathrm{IC}_{50}$ values for VCR and 5-Fu were noted for SGC7901-MGr1 cells after adhesion to either ECM components or control ( $p<0.05$, Fig. 5A). Decreased ADR accumulation and retention, and increased release indices ( $<<0.05$, Fig. $5 B$ ), were observed, along with decreased AI values ( $p<0.05$, Fig. $5 C)$, increased expression of P-gp, MRP and Bcl-2, and decreased expression of $B a x(p<0.05$, Fig. 5D and E), all relative to SGC7901 and SGC7901-pc 

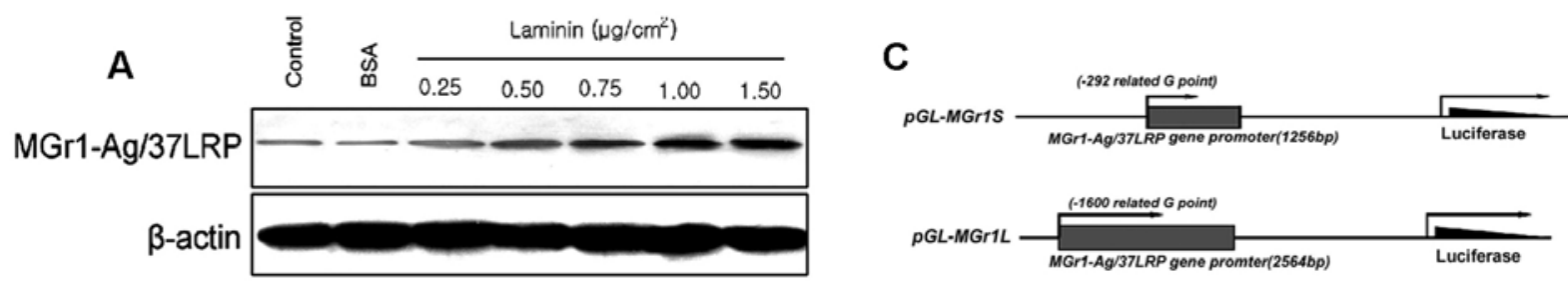

B
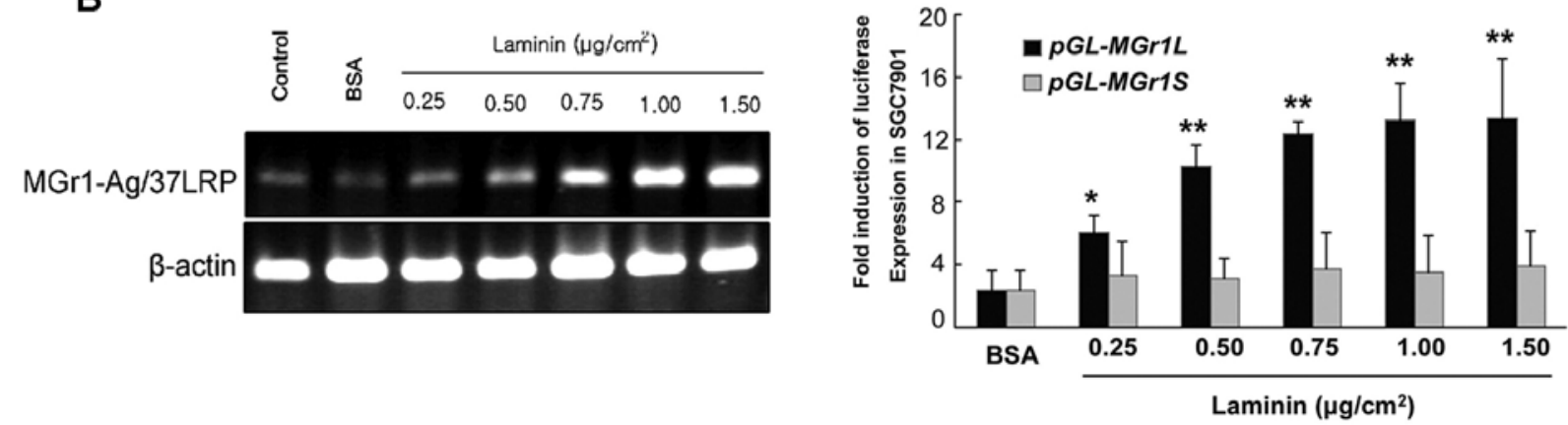

Figure 3. LN adhesion induces upregulation of MGr1-Ag/37LRP in gastric cancer SGC7901 cells. (A and B) LN increased expression of MGr1-Ag/37LRP in SGC7901 cells evaluated by western blotting and RT-PCR. (C) SGC7901 cells were transfected with pGL-MGr1L, pGL-MGr1S or PRL-TK. Luciferase activity results are the means $\pm \mathrm{SE}$ of 3 independent experiments performed in triplicate. ${ }^{*} \mathrm{p}<0.05,{ }^{* *} \mathrm{p}<0.01$ vs. gastric cancer cells adhering to BSA control. $\mathrm{LN}$, laminin; BSA, bovine serum albumin.
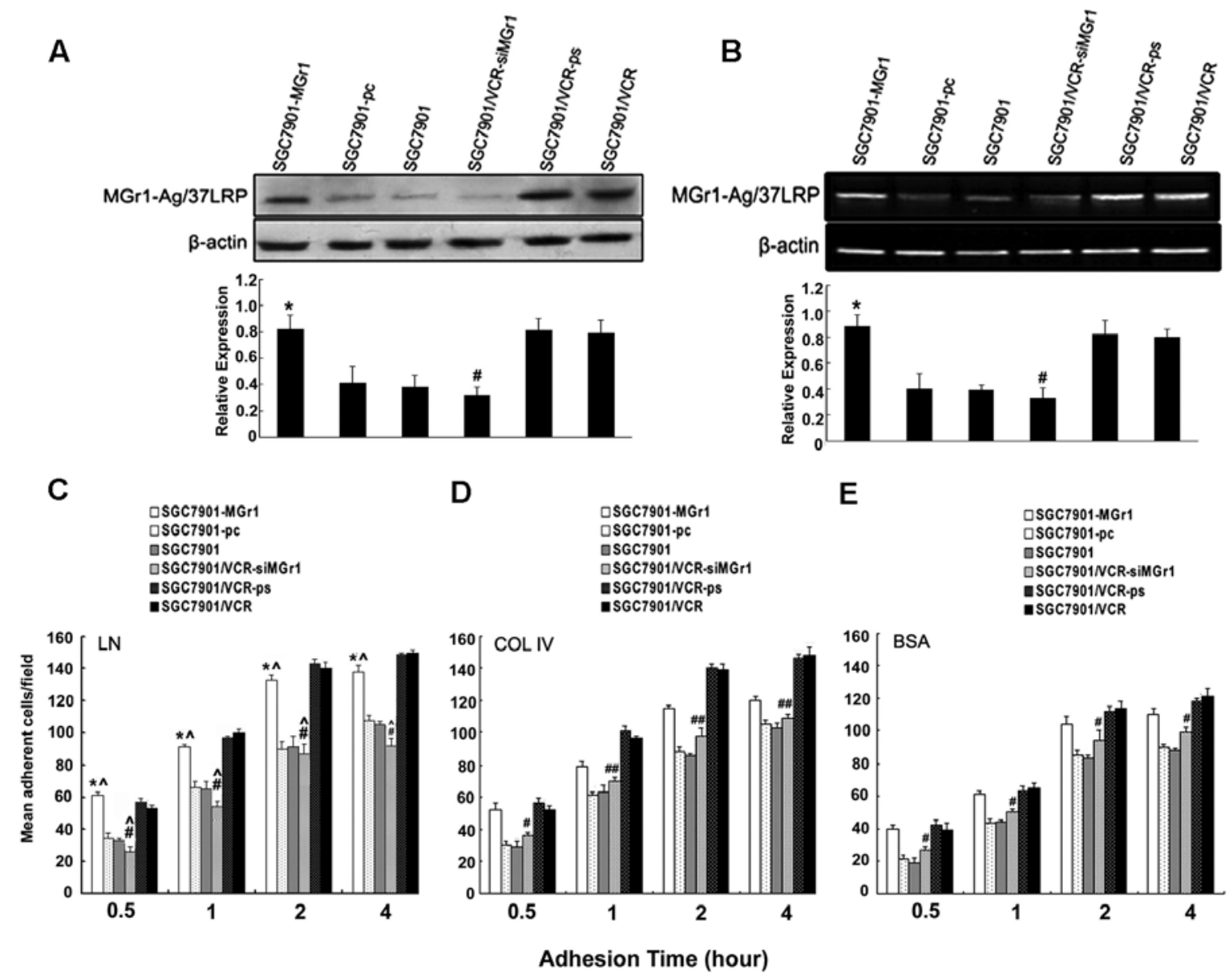

Figure 4. Ability of transfected gastric cancer cells to upregulate or downregulate MGr1-Ag/37LRP after adhesion to ECM components. (A and B) mRNA or proteins from SGC7901/VCR-siMGr1, SGC7901/VCR-ps, SGC7901/VCR, SGC7901-MGr1, SGC7901-pc or SGC7901 cells were analyzed by RT-PCR or western blotting. " p<0.05 vs. SGC7901 and SGC7901-pc. " p<0.05 vs. SGC7901/VCR and SGC7901/VCR-ps. (C-E) Cells attached to ECM components or BSA were counted microscopically after $0.5,1,2$ or $4 \mathrm{~h}$ of adhesion. ${ }^{*} \mathrm{p}<0.05$ vs. SGC7901 and SGC7901-pc adhering to ECM components or BSA control. ${ }^{\# p}<0.05$ or ${ }^{\# \#} \mathrm{p}<0.01 \mathrm{vs.} \mathrm{SGC7901/VCR}$ and SGC7901/VCR-ps adhering to ECM components and BSA control. ${ }^{\wedge} \mathrm{p}<0.05$ vs. SGC7901-MGr1 adhering to COL IV. ECM, extracellular matrix; BSA, bovine serum albumin; COL IV, collagen IV. 

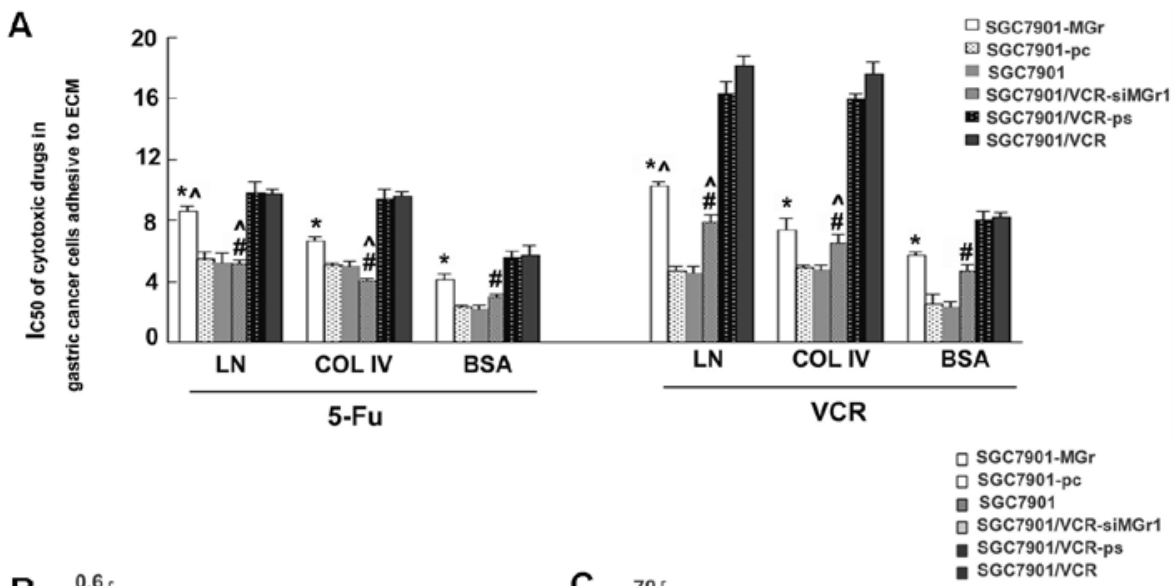

B

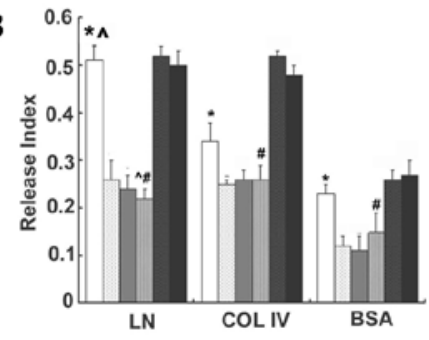

C

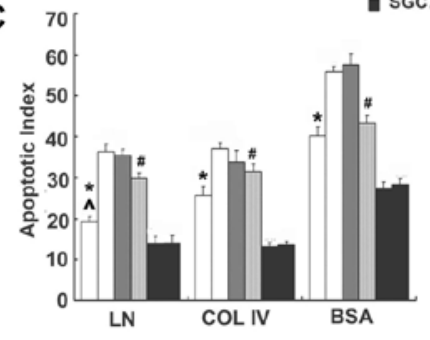

COL IV

BSA

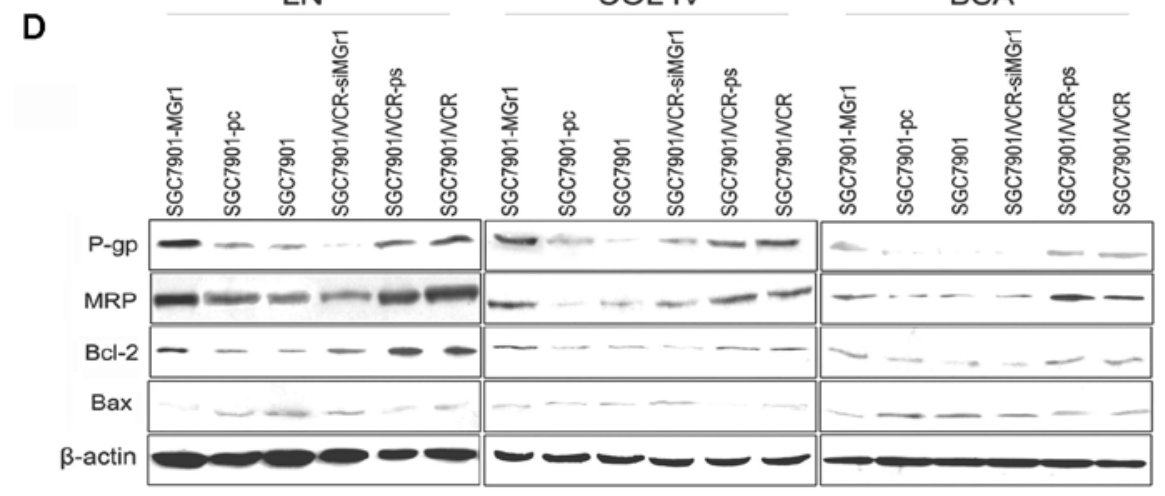

E
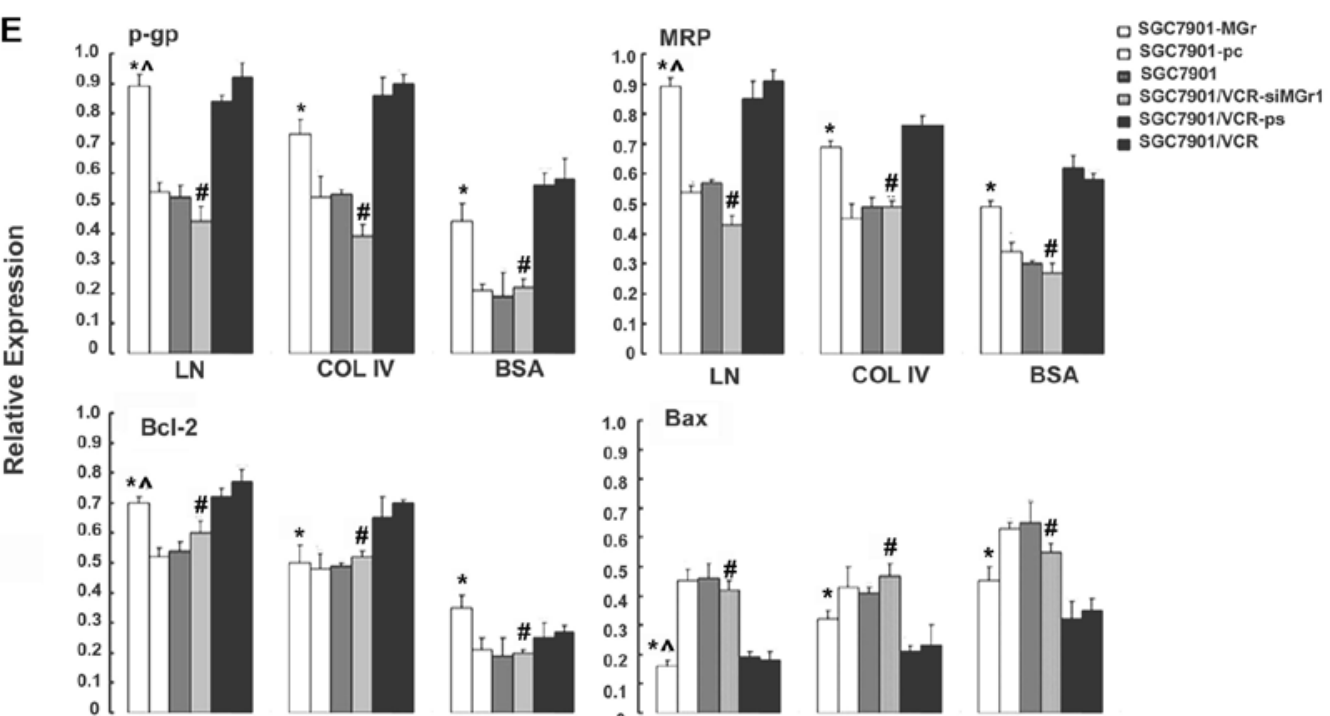

LN

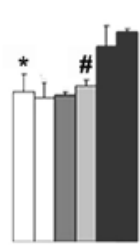

COL IV

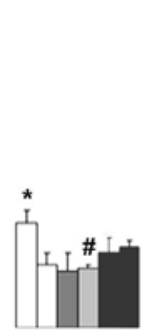

BSA

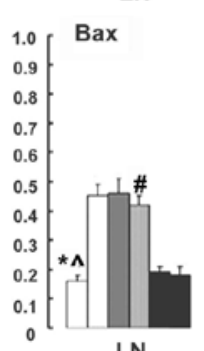

LN

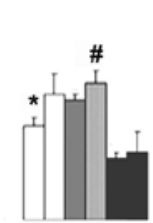

COL IV

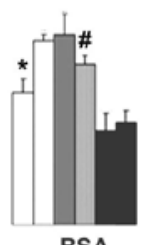

BSA

Figure 5. Characterization of the MDR phenotype of gastric cancer cells transfected to upregulate or downregulate $\mathrm{MGr1}-\mathrm{Ag} / 37 \mathrm{LRP}$ following adhesion to ECM components. (A) Sensitivity of the transfected gastric cancer cell lines to chemotherapeutic drugs, evaluated using colony-forming assay and shown as $\mathrm{IC}_{50}$. (B) ADR release indices of gastric cancer cells calculated by ADR accumulation and retention, as detected by flow cytometry. (C) Apoptotic indices of the gastric cancer cells treated by VCR, as detected by flow cytometry. (D) Expression of P-gp, MRP, Bcl-2 and Bax, evaluated by western blotting with $\beta$-actin as an internal control. (E) Relative expression of P-gp, MRP, Bcl-2 and Bax. Signals were quantified by densitometric scanning. Data are expression relative to $\beta$-actin. " $\mathrm{p}<0.05$ vs. SGC7901 and SGC7901-pc adhering to ECM components and BSA control. ${ }^{*} \mathrm{p}<0.05$ vs. SGC7901/VCR and SGC7901/VCR-ps adhering to ECM components and BSA control. ^ p<0.05 vs. SGC7901-MGr1 adhering to COL IV. ECM, extracellular matrix; ADR, adriamycin; P-gp, P-glycoprotein; MRP, multidrug resistance-associated protein; BSA, bovine serum albumin; COL IV, collagen IV. 


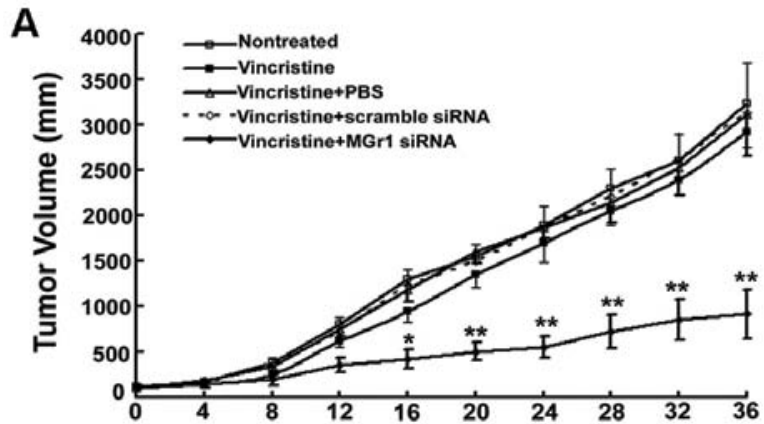

C

B
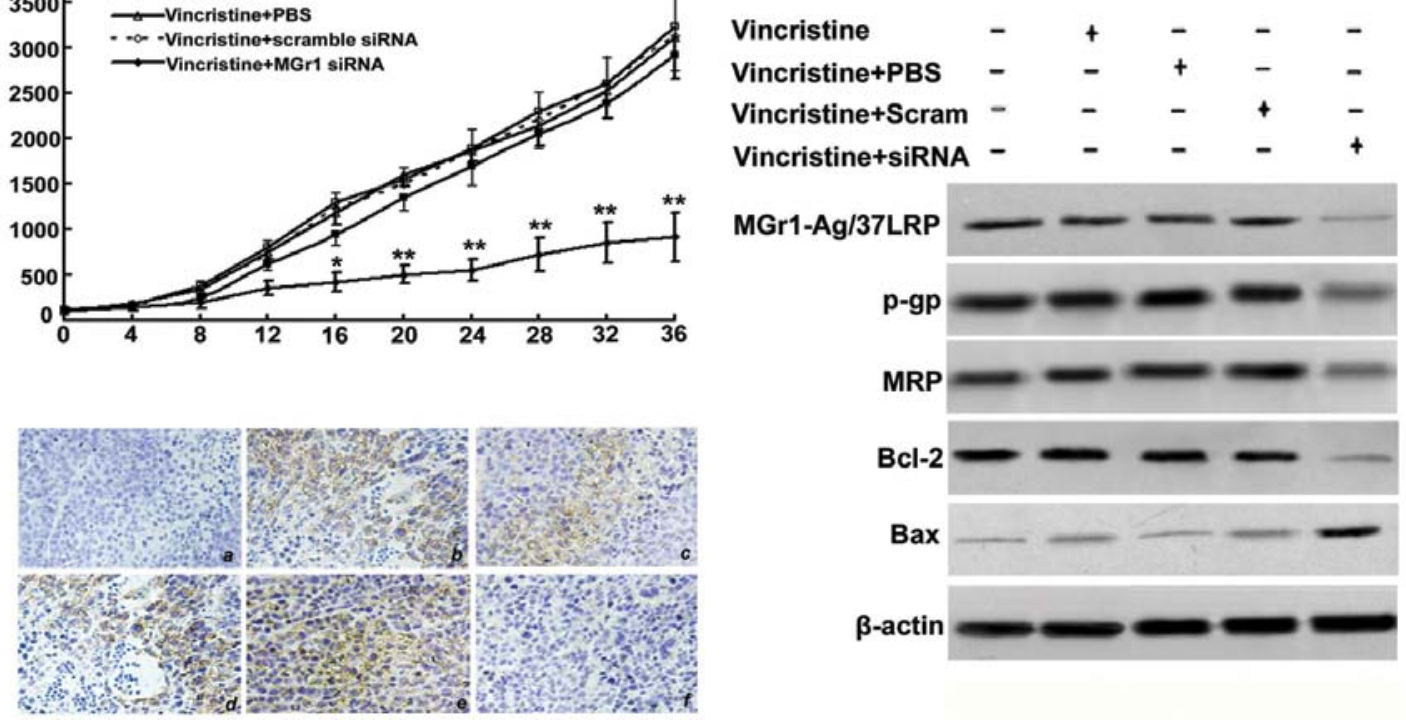

Figure 6. Effect of MGr1-Ag/37LRP siRNA on SGC7901/VCR tumor growth and chemosensitivity in vivo. (A) Mice bearing SGC7901/VCR cell-derived tumors were randomly selected for different treatments (see details in Materials and methods). ${ }^{p} \mathrm{p}<0.05$ compared to control. (B) Immunohistochemical staining for MGr1-Ag/37LRP in paraffin-embedded sections from subcutaneous tumors: (a) negative control group (non-treated tumor with mouse IgG staining); (b) non-treatment with VCR group; (c) VCR treatment group; (d) VCR treatment plus scrambled siRNA group; (e) positive control group (nontreatment tumor); (f) VCR treatment plus MGr1-Ag siRNA group (original magnification, x200). (C) Protein expression of MGr1-Ag/37LRP, P-gp, MRP, Bax and Bcl-2 in vivo. MGrl-Ag/37LRP, P-gp, MRP, Bax, Bcl-2 and $\beta$-actin were assessed by western blotting. P-gp, P-glycoprotein; MRP, multidrug resistance-associated protein.

cells under the same conditions. SGC7901/VCR-siMGr1 cells showed significantly decreased $\mathrm{IC}_{50}$ values for VCR and 5-Fu, decreased release indices, increased AI values, decreased expression of P-gp, MRP and Bcl-2, and increased expression of Bax compared to SGC7901/VCR and SGC7901/VCR-ps cells under the same conditions $(\mathrm{p}<0.05)$. In addition, after adhesion to LN, SGC7901-MGr1 cells showed significantly increased VCR and 5-Fu $\mathrm{IC}_{50}$ values, increased release indices, decreased AI values, increased expression of P-gp, MRP and Bcl-2, and decreased expression of Bax compared to the same cells after COL IV adhesion $(\mathrm{p}<0.05)$. These data indicated that overexpression of MGrl-Ag/37LRP partially promoted the MDR phenotype of gastric cancer cells by decreasing intracellular drug accumulation and inhibiting drug-induced apoptosis.

siRNA of MGrl-Ag/37LRP partly reverses the MDR phenotype of SGC7901/VCR cells in vivo. To address the potential effects of MGr1-Ag/37LRP siRNA in vivo, equal numbers of MGr1-Ag/37LRP siRNA, scrambled sequence oligonucleotide, or PBS with VCR were injected into nude mouse subcutaneously transplanted tumors derived from SGC7901/ VCR cell lines. As shown in Fig. 6A, MGr1-Ag/37LRP siRNA with VCR monotherapy significantly reduced SGC7901/VCR tumor volume by $50 \%$ from days 12 to 36 , relative to treatment with PBS alone, VCR alone, VCR plus scrambled sequence, or the untreated control $(\mathrm{p}<0.01)$. We examined expression levels of MGr1-Ag/37LRP, the drug transporter proteins P-pg and MRP1 and the apoptosis-related proteins Bcl-2 and Bax in the transplanted tumor tissues. Immunohistochemistry staining and western blot analysis were performed on lysates from tumor tissues after MGr1-Ag/37LRP siRNA plus VCR, scrambled siRNA plus VCR, VCR or PBS alone. A marked decrease in expression of MGrl-Ag/37LRP protein was observed in the SGC7901/VCR tumors treated with MGr1-Ag/37LRP siRNA compared to the four controls (Fig. 6B and C). Western blot analyses showed that blocking MGr1-Ag/37LRP expression with siRNA significantly decreased Bcl-2, P-gp and MRP1 expression, while increasing expression of pro-apoptotic Bax (Fig. 6C).

\section{Discussion}

Emerging evidence has shown that the main mechanism underlying drug resistance in myeloma cells is adhesion to the ECM $(19,20)$. In the present study, we present initial evidence that the laminin receptor MGr1-Ag/37LRP increased the adhesive ability of gastric cancer cells to the ECM, subsequently leading to CAM-DR.

The ECM is a complicated network of multifunctional molecules that provides a sophisticated microenvironment for cell survival, metabolism, migration, proliferation and differentiation $(21,22)$. LN and COL IV are the major components of the basement membrane, and are implicated in carcinogenesis and progression in gastric cancer cells (23). In the present study, we concluded that the adhesive ability of MDR gastric cancer cells was significantly increased over the parental cells that were sensitive to chemotherapeutic drugs. After adhesion to the ECM components LN or COL IV, resistance to VCR and ADR increased, suggesting that the MDR phenotype of gastric cancer cells was associated with the cell adhesion state. We also found that exogenous 
overexpression of MGr1-Ag/37LRP upregulated the adhesive ability and drug resistance of both drug-sensitive and MDR gastric cancer cell lines. Stably transfected gastric cancer cells (SGC7901-MGr1) that overexpressed MGr1-Ag/37LRP had a higher ability to adhere to ECM components, and a greater resistance to chemotherapeutic drugs. The ability of SGC7901-MGr1 cells to adhere to LN was stronger than adherence to COL IV, and binding of MGr1-Ag/37LRP to LN may be associated with gastric cancer cell adhesion. The drug resistance of SGC7901-MGrl cells adhering to LN was significantly increased over cells adhering to COL IV, thus binding of MGr1-Ag/37LRP to LN may be involved in CAM-DR in gastric cancer. Further study indicated that binding of MGr1-Ag/37LRP to LN promoted the CAM-DR phenotype of gastric cancer cells by decreasing intracellular drug accumulation, and inhibiting drug-induced apoptosis through regulation of $\mathrm{P}-\mathrm{gp}, \mathrm{MRP}, \mathrm{Bcl}-2$ and Bax expression. These results indicated that the binding of MGr1-Ag/37LRP to its ligand LN upregulated two major drug transporters, and regulated two apoptosis-related molecules, leading to the chemoresistance in gastric cancer cells. Notably, we found that MGr1-Ag/37LRP protein and mRNA levels were upregulated significantly in gastric cancer cells adhering to LN. Dual luciferase assays showed increased transcription of the MGr1 promoter (-1600 and -292) in SGC7901 cells adhering to LN. We propose that MGr1-Ag/37LRP aggravates MDR with the binding to its ligand LN, at the same time, LN induces MGr1Ag/37LRP expression by enhancing transcription. The above data suggest that MGr1-Ag/37LRP interaction with laminin induces CAM-DR which may be reinforced by MGr1-LN binding by a positive feedback loop.

In vivo, siRNA of MGr1-Ag/37LRP significantly enhanced xenograft sensitivity to chemotherapeutic drugs, decreasing the volume of transplanted tumors. The expression of P-gp, MRP, Bcl-2 and MGr1-Ag/37LRP in the xenograft tissues decreased significantly after treatment with MGrl-Ag siRNA, while pro-apoptotic Bax increased.

In conclusion, the present study revealed that binding of MGr1-Ag/37LRP with laminin conferred CAM-DR in gastric cancer by decreasing intracellular drug accumulation and by inhibiting drug-induced apoptosis. Regulation of P-gp, MRP and apoptosis-relate genes (Bcl-2 and Bax expression) may be the underlying mechanisms, and a positive feedback of MGr1$\mathrm{Ag} / 37 \mathrm{LRP}$ transcription may be involved. Inhibition of the expression of MGr1-Ag/37LRP enhanced sensitivity of gastric cancer cells to chemotherapeutic drugs both in vitro and in vivo. MGr1-Ag/37LRP may serve as an effective potential target for reversing the MDR of gastric cancer. However, further studies are needed before a final conclusion is drawn. The present study was based on a single type of cancer cell line. The MDR gastric cancer cells and their parental cells were well characterized and rigorously studied, which provided an ideal cell model. Although well-established drug-resistant cells of other tissue origins may not be available, the findings of this study need to be verified using other types of cancers. In addition, how interaction of MGrl-Ag/37LRP with cell adhesion ligands regulates the expression of various genes remains to be clarified. Various pathways have been implicated in adhesioninduced apoptosis resistance in cancer including MAPK (mitogen-activated protein kinase)/ERK (extracellular regu- lated kinase) kinase (MEK) and phosphatidylinositol-3-kinase (PI3-K)-mediated survival cascades (24). The regulatory mechanisms of gene expression by MGr1-Ag/37LRP warrant further research.

\section{Acknowledgements}

This study was supported by the National Nature Science Foundation of China (no. 81272349), and the 973 National Key Scientific Research Project (no. 2010CB732400).

\section{References}

1. Fan DM, Xiao B, Shi YQ, Ming-Feng, Qiao TD, Chen BJ and Chen Z: A novel cDNA fragment associated with gastric cancer drug resistance was screened out from a library by monoclonal antibody MGr1. World J Gastroenterol 4: 110-111, 1998.

2. Shi Y, Zhai H, Wang X, et al: Multidrug-resistance-associated protein MGr1-Ag is identical to the human 37-kDa laminin receptor precursor. Cell Mol Life Sci 59: 1577-1583, 2002.

3. Sun L, Shi Y, Guo C, et al: Regulation of multidrug resistance by MGr1-antigen in gastric cancer cells. Tumour Biol 27: 27-35, 2006.

4. Boudreau NJ and Jones PL: Extracellular matrix and integrin signalling: the shape of things to come. Biochem J 339: 481-488, 1999.

5. Damiano JS, Cress AE, Hazlehurst LA, Shtil AA and Dalton WS: Cell adhesion mediated drug resistance (CAM-DR): role of integrins and resistance to apoptosis in human myeloma cell lines. Blood 93: 1658-1667, 1999.

6. Nakamura K, Mori M and Enjoji M: Distribution of basement membrane antigens in clinical gastric adenocarcinomas: an immunohistochemical study. J Clin Pathol 40: 1418-1423, 1987.

7. Kawaguchi M, Akagi M, Gray MJ, Liu W, Fan F and Ellis LM: Regulation of vascular endothelial growth factor expression in human gastric cancer cells by interleukin-1 $\beta$. Surgery 136: 686-692, 2004.

8. Jaseja M, Mergen L, Gillette K, Forbes K, Sehgal I and Copié V: Structure-function studies of the functional and binding epitope of the human $37 \mathrm{kDa}$ laminin receptor precursor protein. J Pept Res 66: 9-18, 2005

9. Cai X, Zhang X and Fan D: Establishment of multidrug resistant gastric cancer cell line and its biological characteristics. Chin J Clin Oncol 2S: 67-71, 1994 (in Chinese with English abstract).

10. Liu L, Zhang H, Sun L, et al: ERK/MAPK activation involves hypoxia-induced MGrl-Ag/37LRP expression and contributes to apoptosis resistance in gastric cancer. Int J Cancer 127: 820-829, 2010.

11. Liu L, Sun L, Zhang H, et al: Hypoxia-mediated up-regulation of MGr1-Ag/37LRP in gastric cancers occurs via hypoxiainducible-factor 1-dependent mechanism and contributes to drug resistance. Int J Cancer 124: 1707-1715, 2009.

12. Fan K, Fan D, Cheng LF and Li C: Expression of multidrug resistance-related markers in gastric cancer. Anticancer Res 20: 4809-4814, 2000.

13. Thamilselvan V and Basson MD: Pressure activates colon cancer cell adhesion by inside-out focal adhesion complex and actin cytoskeletal signaling. Gastroenterology 126: 8-18, 2004.

14. Li J, Xu LZ, He KL, et al: Reversal effects of nomegestrol acetate on multidrug resistance in adriamycin-resistant MCF7 breast cancer cell line. Breast Cancer Res 3: 253-263, 2001.

15. Filleur S, Courtin A, Ait-Si-Ali S, et al: SiRNA-mediated inhibition of vascular endothelial growth factor severely limits tumor resistance to antiangiogenic thrombospondin-1 and slows tumor vascularization and growth. Cancer Res 63: 3919-3922, 2003.

16. Kuo CC, Hsieh HP, Pan WY, et al: BPR0L075, a novel synthetic indole compound with antimitotic activity in human cancer cells, exerts effective antitumoral activity in vivo. Cancer Res 64: 4621-4628, 2004.

17. Gleave M, Tolcher A, Miyake H, et al: Progression to androgen independence is delayed by adjuvant treatment with antisense Bcl-2 oligodeoxynucleotides after castration in the LNCaP prostate tumor model. Clin Cancer Res 5: 2891-2898, 1999. 
18. Romanov VI, Wrathall LS, Simmons TD, Pinto da Silva P and Sobel ME: Protein synthesis is required for laminin-induced expression of the $67-\mathrm{kDa}$ laminin receptor and its $37-\mathrm{kDa}$ precursor. Biochem Biophys Res Commun 208: 637-643, 1995.

19. Noborio-Hatano K, Kikuchi J, Takatoku M, et al: Bortezomib overcomes cell-adhesion-mediated drug resistance through downregulation of VLA-4 expression in multiple myeloma. Oncogene 28: 231-242, 2009.

20. Kobune M, Chiba H, Kato J, et al: Wnt3/RhoA/ROCK signaling pathway is involved in adhesion-mediated drug resistance of multiple myeloma in an autocrine mechanism. Mol Cancer Ther 6: 1774-1784, 2007.
21. Hohenester E and Engel J: Domain structure and organisation in extracellular matrix proteins. Matrix Biol 21: 115-128, 2002.

22. Timpl R: Macromolecular organization of basement membranes. Curr Opin Cell Biol 8: 618-624, 1996.

23. David L, Nesland JM, Holm R and Sobrinho-Simões M: Expression of laminin, collagen IV, fibronectin, and type IV collagenase in gastric carcinoma. An immunohistochemical study of 87 patients. Cancer 73: 518-527, 1994.

24. Westhoff MA and Fulda S: Adhesion-mediated apoptosis resistance in cancer. Drug Resist Updat 12: 127-136, 2009. 